\title{
Malignancy Risk Stratification of Thyroid Nodules According to Echotexture and Degree of Hypoechogenicity: A Multicenter Validation Study
}

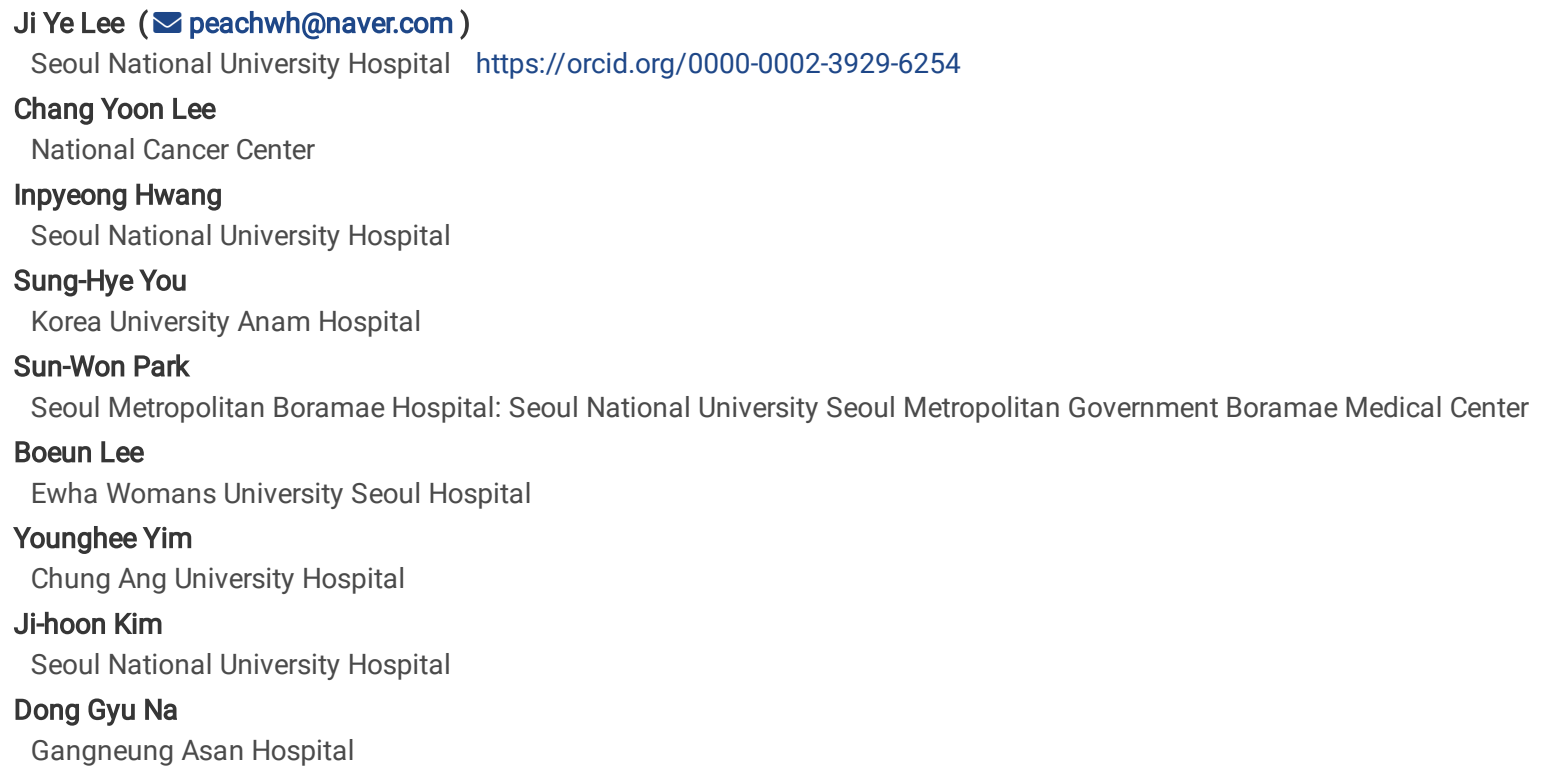

\section{Research Article}

Keywords: Thyroid nodule, Thyroid cancer, Ultrasonography, Fine-needle aspiration, Biopsy

Posted Date: January 6th, 2022

DOI: https://doi.org/10.21203/rs.3.rs-1206756/v1

License: (c) (i) This work is licensed under a Creative Commons Attribution 4.0 International License. Read Full License 


\section{Abstract}

Purpose Various risk stratification systems show some discrepancies in the ultrasound (US) lexicon of nodule echotexture and hypoechogenicity. This study aimed to determine the malignancy risk of thyroid nodules according to their echotexture and degree of hypoechogenicity in a multicenter dataset.

Methods From June to September 2015, 5,601 thyroid nodules ( $\geq 1.0 \mathrm{~cm}$ ) in 4,989 consecutive patients who underwent thyroid US and with a final diagnoses from 26 institutions were evaluated. Thyroid nodules were stratified according to the US echotexture (homogeneous vs. heterogeneous) and degree of hypoechogenicity (mild, moderate, or marked). We then calculated malignancy risk and compared four subgroups stratified with nodule composition and presence of suspicious features.

Results Heterogeneous hypoechoic nodules showed a significantly higher malignancy risk than heterogeneous isoechoic nodules $(P \leq 0.017)$ except in partially cystic nodules. There was no significant difference in malignancy risk between homogeneous vs. heterogeneous hypoechoic nodules ( $P \geq 0.086)$ and between homogeneous vs. heterogeneous iso- or hyperechoic nodules $(P \geq 0.05)$ except in partially cystic nodules without suspicious features. The malignancy risks of both homogeneous and heterogeneous iso- or hyperechoic nodules were low to intermediate, depending on the presence of suspicious features. Regarding the degree of hypoechogenicity, there was no significant difference in malignancy risk between markedly and moderately hypoechoic nodules in all subgroups $(P \geq 0.48)$. Marked or moderately hypoechoic nodules showed a significantly higher risk than mild hypoechoic $(P \leq 0.016)$ and isoor hyperechoic $(P<.001)$ nodules.

Conclusions The predominant echogenicity effectively stratifies the malignancy risk of nodules with heterogeneous echotexture. For malignancy risk stratification, the degree of nodules' hypoechogenicity could be grouped as mild vs. moderate to marked hypoechogenicity.

\section{Introduction}

The echogenicity of a thyroid nodule on ultrasonography (US) is an important descriptor for distinguishing malignancy from benign nodules [1-4]. Previous studies have consistently reported that the malignancy risk of hypoechoic nodules was higher than that of iso- or hyperechoic nodules [1, 3-5]. Marked hypoechogenicity is related to increased malignancy risk of thyroid nodules [1, 5-8] and has been adopted in several risk stratification systems (RSS) [9-12].

Despite of its importance, the US definition of nodule echogenicity shows discrepancies across risk stratification systems (RSS) [13]. For nodules with heterogeneous echogenicity, guidelines adopt different strategies; the Korean Thyroid Imaging Reporting and Data System (K-TIRADS) and American College of Radiology (ACR) TIRADS classified them based on the predominant echogenicity [11, 14], but the European Thyroid Imaging Reporting and Data System (EU-TIRADS) classified heterogeneous nodules as mildly hypoechoic nodules when they had any hypoechoic portion [10]. While the American Association of Clinical Endocrinologists/American College of Endocrinology/Associazione Medici Endocrinologi [9], ACR [11], EU-TIRADS [10], and the Chinese Thyroid Imaging Reporting and Data System (C-TIRADS) [12] distinguished between marked and mild hypoechogenicity, the American Thyroid Association [15] and K-TIRADS [14] did not discriminate between marked versus mild hypoechogenicity regarding nodule risk stratification. In these RSSs, nodules with similar echogenicity to the anterior neck muscles (i.e., moderate hypoechogenicity) were grouped with nodules with mild hypoechogenicity [9-12, 14, 15].

A recent study demonstrated that nodule hypoechogenicity could be stratified as mild versus moderate to marked, and nodules with heterogeneous echogenicity are stratified by the predominant echogenicity of the solid portion [7]. According to the results of this study, the $2021 \mathrm{~K}-\mathrm{TIRADS}$ revised the definition of marked hypoechogenicity as hypoechoic or similar echogenicity relative to the anterior neck muscles [16]. However, insufficient evidence supports this take on hypoechoic nodules' stratification because the previous study was a single-center study [7]. We designed a multicenter study to validate if this revised definition of nodule hypoechogenicity could effectively stratify the malignancy risk of thyroid nodules. The purpose of this study was to validate thyroid nodules' malignancy risk according to their echotexture and degree of hypoechogenicity in a multicenter cohort.

\section{Materials And Methods}

The institutional review boards of the 26 participating centers approved this study. The informed consent requirement was waived for this retrospective review.

\section{Study population}

Patient data were collected from 26 different hospitals in Korea (Thyroid Imaging Network of Korea registry, THINK). From June to September 2015, 22,775 patients underwent thyroid US at the 26 participating institutions. Among them, 16,679 were excluded due to a lack of reference standard test (biopsy or surgery) ( $n=4304$ ), thyroid nodules $<1.0 \mathrm{~cm}$ (maximal diameter, $n=12,130)$, suboptimal image quality $(n=245)$ or inconclusive biopsy results ( $n=1,015$ patients with 1,102 nodules) $[17,18]$. In this study, 59 isolated macrocalcifications and 48 purely cystic nodules were further excluded because of an inability to assess nodule echogenicity. We included a total of 5,601 thyroid nodules in 4,989 patients in this study. (4,101 women, 888 men; age range: $19-76$ years) (Fig. 1). Malignant nodules were diagnosed based on the histopathological results after surgery $(n=927)$ or malignant fine needle aspiration (FNA) or coreneedle biopsy $(C N B)$ results $(n=162)$. Benign nodules were diagnosed based on the histopathological results after surgery $(n=390)$, with at least two benign FNA or CNB results $(n=594)$, and one benign FNA or CNB result $(n=3,528)$.

\section{US examination and image analysis}

All US examinations were performed with a 10-14 MHz linear probe. US images were retrospectively reviewed by one of 17 experienced radiologists with 822 years of experience performing thyroid US using an online program (AIM AiCRO; http://study.aim-aicro.com). Prior to the multicenter study's commencement, we held training sessions to establish a baseline consensus regarding US criteria $[9-11,14,17,18]$. The 17 radiologists evaluated images

Page 2/11 
of biopsy-proven masses not included in the study, and were asked to assess the US criteria during a consensus meeting, including composition, echogenicity, margin, calcification, orientation (taller-than-wide), spongiform appearance, and intracystic echogenic foci with comet-tail artifact. All of the reviewers, who were blind to the FNA results and final diagnoses, then assessed the US features of the thyroid nodules. Reviewers determined the nodule hypoechogenicity by assessing the echogenicity of the solid component in a nodule. In the $2021 \mathrm{~K}$-TIRADS, the nodule was defined as hypoechoic if it was hypoechoic relative to the normal thyroid parenchyma. The echotexture of the nodule was categorized as homogenous or heterogeneous echotexture based on the uniformity of nodule echogenicity [7] (Figure 2). Heterogeneous echotexture was defined as the nodule's solid component showing two obviously different portions of echogenicity (iso- or hyperechoic vs. hypoechogenicity). Heterogeneously echotextured nodules' echogenicity was determined by their predominant echogenicity. The nodules were classified into four groups: homogeneous hypoechoic, heterogeneous hypoechoic, heterogeneous iso- or hyperechoic, and homogeneous iso- or hyperechoic.

The nodule's degree of hypoechogenicity was categorized as mild (hypoechoic relative to the thyroid parenchyma, but hyperechoic relative to the anterior neck muscles), moderate (similar echogenicity to the anterior neck muscles), and marked (hypoechoic relative to the anterior neck muscles) (Figure 3) [5, 7]. We assessed other US features regarding composition and presence of suspicious US features (punctuate echogenic foci, nonparallel orientation, irregular margin) of the thyroid nodules on the basis of the $2021 \mathrm{~K}$-TIRADS [16]. In the $2021 \mathrm{~K}$-TIRADS, punctate echogenic foci were defined as punctate ( $\leq 1 \mathrm{~mm}$ ) hyperechoic foci within the solid component of a nodule, nonparallel orientation as the anteroposterior diameter of a nodule being longer than its transverse diameter in the transverse plane, and irregular margin as a non-smooth edge with spiculation or microlobulation. The suggested malignancy risk in the 2021 K-TIRADS is proposed as follows [16]: high suspicion, > 60\%; intermediate suspicion, $10-40 \%$; low suspicion $3-10 \%$; and benign, $<3 \%$.

\section{Data analysis and statistical analysis}

We calculated each nodule category's frequency and malignancy risk based on its echotexture and degree of hypoechogenicity. Chi-squared or Fisher's exact tests were used to compare the malignancy risk among each group. We performed subgroup analysis to assess the difference between the malignancy risks of thyroid nodules according to their composition (solid vs. partially cystic), and the presence of any suspicious US features. This reflects that the thyroid nodules' malignancy risks differ according to their composition, echogenicity, and presence of suspicious US features [4]. Chi-squared or Fisher's exact tests were used to compare the malignancy risk between homogeneous hypoechoic, heterogeneous hypoechoic, heterogeneous iso- or hyperechoic nodule, and homogeneous iso- or hyperechoic nodules. Statistical analyses were performed using IBM SPSS Statistics for Windows, Version 24.0 (IBM Corp.), and MedCalc $®$ (version 20.009 (MedCalc Software Ltd)). A $P$ value $<0.05$ was considered statistically significant.

\section{Results}

\section{Demographic and clinicopathologic characteristics}

The demographic data are summarized in Table 1. The mean size of the nodules was $53.4 \pm 12.7 \mathrm{~mm}$ (range, 10 to $100 \mathrm{~mm}$ ). Of the 5,601 thyroid nodules, 4,512 (80.6\%) were diagnosed as benign and 1,089 (19.4\%) as malignant. The 1,089 malignant nodules included 989 papillary thyroid carcinomas ( $90.8 \%)$, 62 follicular carcinomas (5.7\%), $12(1.1 \%)$ medullary carcinomas, 7 (0.6\%) poorly differentiated carcinomas, 6 ( $0.6 \%)$ anaplastic carcinomas, 5 ( $0.5 \%)$ metastases, $4(0.4 \%)$ unspecified malignancies, $3(0.3 \%)$ lymphomas, and $1(0.1 \%)$ squamous cell carcinoma. Patients with malignant nodules were significantly younger $(P<.001)$, included smaller proportions of female patients $(P<.001)$, and smaller nodule size $(P<.001)$ than patients with benign nodules. 
Table 1

Demographic Data of 5601 Nodules in This Study

\begin{tabular}{|lllll|}
\hline Nodules & Total (N=561) & Benign (N=4512) & Malignant (N=1089) & $P$ \\
\hline Number of patients & 4989 & 3991 & 998 & $<.001$ \\
\hline Age (years) & $53.4 \pm 12.7$ & $54.6 \pm 13.1$ & $48.8 \pm 13.9$ & $<.001$ \\
\hline No. of female Patients (\%) & $4627(82.6 \%)$ & $3793(84.1 \%)$ & $834(76.6 \%)$ & $<.001$ \\
\hline Mean maximal nodule size (mm) & $20.7 \pm 10.8$ & $21.1 \pm 10.7$ & $19.1 \pm 11.1$ & $<.001$ \\
\hline US echotexture of nodules & & & $782(71.8 \%)$ & $<.001$ \\
\hline Homogeneous & $4274(76.3 \%)$ & $3492(77.4 \%)$ & $307(28.2 \%)$ & \\
\hline Heterogeneous & $1327(23.7 \%)$ & $1020(22.6 \%)$ & \\
\hline US echogenicity of nodules ${ }^{\text {a }}$ & & & $204(18.7 \%)$ & \\
\hline Marked hypoechogenicity & $315(5.6 \%)$ & $111(2.5 \%)$ & $340(31.2 \%)$ & \\
\hline Moderate hypoechogenicity & $705(12.6 \%)$ & $365(8.1 \%)$ & $234(21.5 \%)$ & \\
\hline Mild hypoechogenicity & $994(17.7 \%)$ & $760(16.8 \%)$ & $311(28.6 \%)$ & \\
\hline Iso- or hyperechogenicity & $3587(64.0 \%)$ & $3276(72.6 \%)$ & \\
\hline Unless otherwise indicated, data are numbers with percentages in parentheses for categorical variables
\end{tabular}

When we determined the heterogeneously echotextured nodules' echogenicity by the predominant echogenicity, iso- or hyperechogenicity was the most common (64.0\%) in all nodules. Among 778 hypoechoic malignant nodules, 718 (92.3\%) were PTCs, including 58 (7.5\%) follicular variant PTCs. Thirty (3.9\%) were follicular carcinomas. In 311 iso- or hyperechoic malignant nodules, 271 (87.1\%) were PTCs, including 69 (21.9\%) follicular variant PTCs. Thirty-two (10.3\%) were follicular carcinomas.

\section{Comparison of malignancy risk between nodules with homogeneous and heterogeneous echotexture}

Table 2 shows malignancy risks nodules classified according to their echogenicity and echotexture. Overall, the homogeneous hypoechoic nodules' malignancy risk was significantly higher than heterogeneous hypoechoic nodules ( 40.5 vs. $33.5 \%, P=0.022$ ). Heterogeneous hypoechoic nodules showed significantly higher malignancy risk than heterogeneous iso- or hyperechoic nodules (33.5 vs. $15.8 \%, P<.001)$. Heterogeneous iso- or hyperechoic nodules showed significantly higher malignancy risk than homogeneous iso- or hyperechoic nodules $(15.8$ vs. $6.7 \%, P<.001)$. 
Table 2

Malignancy risk stratified by echogenicity and echotexture

\begin{tabular}{|c|c|c|c|c|c|c|c|c|c|c|}
\hline \multicolumn{5}{|c|}{$A / I$} & \multicolumn{3}{|c|}{ With suspicious features ${ }^{c}$} & \multicolumn{3}{|c|}{ No suspicious US features ${ }^{c}$} \\
\hline Echotexture & Echogenicity & $\begin{array}{l}\text { No of } \\
\text { malignant } \\
\text { nodules, } \\
N(\%)\end{array}$ & $\begin{array}{l}\text { No. of } \\
\text { nodules, } \\
N(\%)\end{array}$ & $\begin{array}{l}\text { Malignancy } \\
\text { risk (\%) }\end{array}$ & $\begin{array}{l}\text { No of } \\
\text { malignant } \\
\text { nodules, } \\
N(\%)\end{array}$ & $\begin{array}{l}\text { No. of } \\
\text { nodules, } \\
N(\%)\end{array}$ & $\begin{array}{l}\text { Malignancy } \\
\text { risk (\%) }\end{array}$ & $\begin{array}{l}\text { No of } \\
\text { malignant } \\
\text { nodules, } \\
N(\%)\end{array}$ & $\begin{array}{l}\text { No. of } \\
\text { nodules, } \\
N(\%)\end{array}$ & $\begin{array}{l}\text { Malignancy } \\
\text { risk (\%) }\end{array}$ \\
\hline \multicolumn{11}{|l|}{ All } \\
\hline \multirow[t]{2}{*}{ Homogeneous } & $\begin{array}{l}\text { Hypoechoic } \\
\text { b }\end{array}$ & $\begin{array}{l}594 \\
(54.5)\end{array}$ & $\begin{array}{l}1465 \\
(26.2)\end{array}$ & 40.5 & $\begin{array}{l}448 \\
(63.1)\end{array}$ & $\begin{array}{l}686 \\
(45.5)\end{array}$ & 65.3 & $\begin{array}{l}146 \\
(38.5)\end{array}$ & $\begin{array}{l}779 \\
(19.0)\end{array}$ & 18.7 \\
\hline & $\begin{array}{l}\text { Iso- or } \\
\text { hyperechoic }\end{array}$ & $\begin{array}{l}188 \\
(17.3)\end{array}$ & $\begin{array}{l}2809 \\
(50.2)\end{array}$ & 6.7 & $66(9.3)$ & $\begin{array}{l}371 \\
(24.6)\end{array}$ & 17.8 & $\begin{array}{l}122 \\
(32.2)\end{array}$ & $\begin{array}{l}2438 \\
(59.6)\end{array}$ & 5.0 \\
\hline \multirow[t]{2}{*}{$\begin{array}{l}\text { a } \\
\text { Heterogeneous }\end{array}$} & $\begin{array}{l}\text { Hypoechoic } \\
\text { b }\end{array}$ & $\begin{array}{l}184 \\
(16.9)\end{array}$ & $\begin{array}{l}549 \\
(9.8)\end{array}$ & 33.5 & $\begin{array}{l}129 \\
(18.2)\end{array}$ & $\begin{array}{l}228 \\
(15.1)\end{array}$ & 56.6 & $55(14.5)$ & $\begin{array}{l}321 \\
(7.8)\end{array}$ & 17.1 \\
\hline & $\begin{array}{l}\text { Iso- or } \\
\text { hyperechoic }\end{array}$ & $\begin{array}{l}123 \\
(11.3)\end{array}$ & $\begin{array}{l}778 \\
(13.9)\end{array}$ & 15.8 & $67(9.4)$ & $\begin{array}{l}223 \\
(14.8)\end{array}$ & 30.0 & $56(14.8)$ & $\begin{array}{l}555 \\
(13.6)\end{array}$ & 10.1 \\
\hline Total & & $\begin{array}{l}1089 \\
(100.0)\end{array}$ & $\begin{array}{l}5601 \\
(100.0)\end{array}$ & 19.4 & $\begin{array}{l}710 \\
(100.0)\end{array}$ & $\begin{array}{l}1508 \\
(100.0)\end{array}$ & 47.1 & $\begin{array}{l}379 \\
(100.0)\end{array}$ & $\begin{array}{l}4093 \\
(100.0)\end{array}$ & 9.3 \\
\hline \multicolumn{11}{|l|}{ Solid } \\
\hline \multirow[t]{2}{*}{ Homogeneous } & $\begin{array}{l}\text { Hypoechoic } \\
\text { b }\end{array}$ & $\begin{array}{l}554 \\
(61.8)\end{array}$ & $\begin{array}{l}1183 \\
(38.9)\end{array}$ & 46.8 & $\begin{array}{l}425 \\
(67.6)\end{array}$ & $\begin{array}{l}611 \\
(56.6)\end{array}$ & 69.6 & $\begin{array}{l}129 \\
(48.1)\end{array}$ & $\begin{array}{l}572 \\
(29.2)\end{array}$ & 22.6 \\
\hline & $\begin{array}{l}\text { Iso- or } \\
\text { hyperechoic }\end{array}$ & $\begin{array}{l}110 \\
(12.3)\end{array}$ & $\begin{array}{l}1044 \\
(34.3)\end{array}$ & 10.5 & $44(7.0)$ & $\begin{array}{l}165 \\
(15.3)\end{array}$ & 26.7 & $66(24.6)$ & $\begin{array}{l}879 \\
(44.8)\end{array}$ & 7.5 \\
\hline \multirow[t]{2}{*}{ a } & $\begin{array}{l}\text { Hypoechoic } \\
\text { b }\end{array}$ & $\begin{array}{l}151 \\
(16.8)\end{array}$ & $\begin{array}{l}377 \\
(12.4)\end{array}$ & 40.1 & $\begin{array}{l}111 \\
(17.6)\end{array}$ & $\begin{array}{l}169 \\
(15.6)\end{array}$ & 65.7 & $40(14.9)$ & $\begin{array}{l}208 \\
(10.6)\end{array}$ & 19.2 \\
\hline & $\begin{array}{l}\text { Iso- or } \\
\text { hyperechoic }\end{array}$ & $82(9.1)$ & $\begin{array}{l}437 \\
(14.4)\end{array}$ & 18.8 & $49(7.8)$ & $\begin{array}{l}135 \\
(12.5)\end{array}$ & 36.3 & $33(12.3)$ & $\begin{array}{l}302 \\
(15.4)\end{array}$ & 10.9 \\
\hline Total & & $\begin{array}{l}897 \\
(100.0)\end{array}$ & $\begin{array}{l}3041 \\
(100.0)\end{array}$ & 29.5 & $\begin{array}{l}629 \\
(100.0)\end{array}$ & $\begin{array}{l}1080 \\
(100.0)\end{array}$ & 58.2 & $\begin{array}{l}268 \\
(100.0)\end{array}$ & $\begin{array}{l}1961 \\
(100.0)\end{array}$ & 13.7 \\
\hline \multicolumn{11}{|l|}{ Partially cystic } \\
\hline \multirow[t]{2}{*}{ Homogeneous } & $\begin{array}{l}\text { Hypoechoic } \\
\text { b }\end{array}$ & $40(20.8)$ & $\begin{array}{l}282 \\
(11.0)\end{array}$ & 14.2 & $23(28.4)$ & $\begin{array}{l}75 \\
(17.5)\end{array}$ & 30.7 & $17(15.3)$ & $\begin{array}{l}207 \\
(9.7)\end{array}$ & 8.2 \\
\hline & $\begin{array}{l}\text { Iso- or } \\
\text { hyperechoic }\end{array}$ & $78(40.6)$ & $\begin{array}{l}1765 \\
(68.9)\end{array}$ & 4.4 & $22(27.2)$ & $\begin{array}{l}206 \\
(48.1)\end{array}$ & 10.7 & $56(50.5)$ & $\begin{array}{l}1559 \\
(73.1)\end{array}$ & 3.6 \\
\hline \multirow[t]{2}{*}{$\begin{array}{l}\text { a } \\
\text { Heterogeneous }\end{array}$} & $\begin{array}{l}\text { Hypoechoic } \\
\text { b }\end{array}$ & $33(17.2)$ & $\begin{array}{l}172 \\
(6.7)\end{array}$ & 19.2 & $18(22.2)$ & $\begin{array}{l}59 \\
(13.8)\end{array}$ & 30.5 & $15(13.5)$ & $\begin{array}{l}113 \\
(5.3)\end{array}$ & 13.3 \\
\hline & $\begin{array}{l}\text { Iso- or } \\
\text { hyperechoic }\end{array}$ & $41(21.4)$ & $\begin{array}{l}341 \\
(13.3)\end{array}$ & 12.0 & $18(22.2)$ & $\begin{array}{l}88 \\
(20.6)\end{array}$ & 20.5 & $23(20.7)$ & $\begin{array}{l}253 \\
(11.9)\end{array}$ & 9.1 \\
\hline Total & & $\begin{array}{l}192 \\
(100.0)\end{array}$ & $\begin{array}{l}2560 \\
(100.0)\end{array}$ & 7.5 & $\begin{array}{l}81 \\
(100.0)\end{array}$ & $\begin{array}{l}428 \\
(100.0)\end{array}$ & 18.9 & $\begin{array}{l}111 \\
(100.0)\end{array}$ & $\begin{array}{l}2132 \\
(100.0)\end{array}$ & 6.2 \\
\hline
\end{tabular}

a Nodule echogenicity was categorized by the predominant echogenicity in nodules with heterogeneous echotexture.

${ }^{\mathrm{b}}$ Hypoechogenicity includes any of marked-, moderate-, and mild hypoechogenicity.

c Suspicious US features include punctuate echogenic foci, nonparallel orientation (taller than wide) and irregular shape.

When we classified the nodules according to composition and presence of suspicious features, there was no significant difference in malignancy risks between homogeneous hypoechoic and heterogeneous hypoechoic nodules in all subgroups $(P \geq 0.086)$. On the contrary, heterogeneous hypoechoic nodules showed significantly higher malignancy risks than heterogeneous isoechoic nodules in all subgroups $(P \leq 0.017)$ except partially cystic nodules. The malignancy risks were not significantly different between heterogeneous iso- or hyperechoic nodules and homogeneous isoechoic nodules in all subgroups except in the partially cystic nodules subgroup without suspicious features $(P \geq 0.05)$.

\section{Risk stratification of thyroid nodules with heterogeneous echotexture}

In terms of risk stratification, the malignancy risks of solid heterogeneous hypoechoic nodules with suspicious features were stratified within the high suspicion category, along with solid homogeneous hypoechoic nodules with suspicious features (65.7\% malignancy risk in solid heterogeneous hypoechoic nodules with suspicious features and $69.6 \%$ in solid homogeneous hypoechoic nodules with suspicious features). The malignancy risks of solid heterogeneous iso- or hyperechoic nodules ranged within the intermediate suspicion category, depending on the presence of suspicious US features (10.9\% in nodules without suspicious features and $36.3 \%$ in nodules with suspicious features). The malignancy risk of solid homogeneous iso- or hyperechoic nodules 
ranged within the low to intermediate suspicion categories, depending on the presence of suspicious US features (7.5\% in solid homogeneous iso- or hyperechoic nodules without suspicious features, $26.7 \%$ in solid homogeneous iso- or hyperechoic nodules with suspicious features).

In partially cystic nodules, the malignancy risks of hypoechoic or iso- or hyperechoic nodules (either homogeneous or heterogeneous) with suspicious features were stratified within the intermediate suspicion category (10.7-30.7\%). The malignancy risks of all partially cystic nodules without suspicious features ranged within the low to intermediate suspicion category (3.6-13.3\%), regardless of echogenicity and echotexture.

\section{Malignancy risk according to degree of predominant hypoechogenicity}

Table 3 lists the calculated malignancy risks of nodules categorized by their predominant degree of hypoechogenicity grouped by overall nodules and subgroups. Overall, markedly hypoechoic nodules demonstrated a significantly higher malignancy risk than moderately $(P<.001)$ and mildly hypoechoic $(P<$ .001) nodules. The malignancy risk of markedly and moderately hypoechoic nodules was significantly higher than that of mildly hypoechoic nodules $(P<$ $.001)$.

Table 3

Malignancy Risk Stratified by Degree of Hypoechogenicity and Predominant Echogenicity According to Composition and suspicious Features

\begin{tabular}{|c|c|c|c|c|c|c|c|c|c|}
\hline \multirow[t]{2}{*}{ Echogenicity } & \multicolumn{3}{|l|}{ All } & \multicolumn{3}{|c|}{ Nodules with suspicious features ${ }^{a}$} & \multicolumn{3}{|c|}{ Nodules without suspicious features ${ }^{\text {a }}$} \\
\hline & $\begin{array}{l}\text { No. of } \\
\text { malignant } \\
\text { nodules, } N \\
(\%)\end{array}$ & $\begin{array}{l}\text { No. of } \\
\text { nodules, } \\
N(\%)\end{array}$ & $\begin{array}{l}\text { Malignancy } \\
\text { risk (\%) }\end{array}$ & $\begin{array}{l}\text { No. of } \\
\text { malignant } \\
\text { nodules, N } \\
(\%)\end{array}$ & $\begin{array}{l}\text { No. of } \\
\text { nodules, } \\
N(\%)\end{array}$ & $\begin{array}{l}\text { Malignancy } \\
\text { risk (\%) }\end{array}$ & $\begin{array}{l}\text { No. of } \\
\text { malignant } \\
\text { nodules, N } \\
(\%)\end{array}$ & $\begin{array}{l}\text { No. of } \\
\text { nodules, } \\
N(\%)\end{array}$ & $\begin{array}{l}\text { Malignancy } \\
\text { risk (\%) }\end{array}$ \\
\hline \multicolumn{10}{|l|}{ All } \\
\hline $\begin{array}{l}\text { Marked } \\
\text { hypoechogenicity }\end{array}$ & 204 (18.7) & $315(5.6)$ & 64.8 & $178(25.1)$ & $\begin{array}{l}228 \\
(15.1)\end{array}$ & 78.1 & $26(6.9)$ & $87(2.1)$ & 29.9 \\
\hline $\begin{array}{l}\text { Moderate } \\
\text { hypoechogenicity }\end{array}$ & 340 (31.2) & $\begin{array}{l}705 \\
(12.6)\end{array}$ & 48.2 & $261(36.8)$ & $\begin{array}{l}370 \\
(24.5)\end{array}$ & 70.5 & $79(20.8)$ & $\begin{array}{l}334 \\
(8.2)\end{array}$ & 23.7 \\
\hline $\begin{array}{l}\text { Mild } \\
\text { hypoechogenicity }\end{array}$ & $234(21.5)$ & $\begin{array}{l}994 \\
(17.7)\end{array}$ & 23.5 & $138(19.4)$ & $\begin{array}{l}316 \\
(21.1)\end{array}$ & 43.7 & $96(25.3)$ & $\begin{array}{l}677 \\
(16.6)\end{array}$ & 14.2 \\
\hline $\begin{array}{l}\text { Iso- or } \\
\text { hyperechogenicity }\end{array}$ & 311 (28.6) & $\begin{array}{l}3587 \\
(64.0)\end{array}$ & 8.7 & $133(18.7)$ & $\begin{array}{l}594 \\
(39.4)\end{array}$ & 22.4 & $177(47.0)$ & $\begin{array}{l}2989 \\
(73.1)\end{array}$ & 5.9 \\
\hline Total & $1089(100.0)$ & $\begin{array}{l}5601 \\
(100.0)\end{array}$ & 19.4 & $710(100.0)$ & $\begin{array}{l}1508 \\
(100.0)\end{array}$ & 47.1 & $378(100.0)$ & $\begin{array}{l}4087 \\
(100.0)\end{array}$ & 9.2 \\
\hline \multicolumn{10}{|l|}{ Solid } \\
\hline $\begin{array}{l}\text { Marked } \\
\text { hypoechogenicity }\end{array}$ & $201(22.4)$ & $\begin{array}{l}304 \\
(10.0)\end{array}$ & 66.1 & $176(28.0)$ & $\begin{array}{l}224 \\
(20.7)\end{array}$ & 78.6 & $25(9.3)$ & $80(4.1)$ & 31.3 \\
\hline $\begin{array}{l}\text { Moderate } \\
\text { hypoechogenicity }\end{array}$ & $312(34.8)$ & $\begin{array}{l}596 \\
(19.6)\end{array}$ & 52.3 & $244(38.8)$ & $\begin{array}{l}333 \\
(30.8)\end{array}$ & 73.3 & $68(25.4)$ & $\begin{array}{l}263 \\
(13.4)\end{array}$ & 25.9 \\
\hline $\begin{array}{l}\text { Mild } \\
\text { hypoechogenicity }\end{array}$ & $192(21.4)$ & $\begin{array}{l}660 \\
(21.7)\end{array}$ & 29.1 & $116(18.4)$ & $\begin{array}{l}223 \\
(20.6)\end{array}$ & 52.0 & $76(28.4)$ & $\begin{array}{l}437 \\
(22.3)\end{array}$ & 17.4 \\
\hline $\begin{array}{l}\text { Iso- or } \\
\text { hyperechogenicity }\end{array}$ & $192(21.4)$ & $\begin{array}{l}1481 \\
(48.7)\end{array}$ & 13.0 & $93(14.8)$ & $\begin{array}{l}300 \\
(27.8)\end{array}$ & 31.0 & $99(36.9)$ & $\begin{array}{l}1181 \\
(60.2)\end{array}$ & 8.4 \\
\hline Total & 897 (100.0) & $\begin{array}{l}3041 \\
(100.0)\end{array}$ & 29.5 & $629(100.0)$ & $\begin{array}{l}1080 \\
(100.0)\end{array}$ & 58.2 & $268(100.0)$ & $\begin{array}{l}1961 \\
(100.0)\end{array}$ & 13.7 \\
\hline \multicolumn{10}{|l|}{ Partially cystic } \\
\hline $\begin{array}{l}\text { Marked } \\
\text { hypoechogenicity }\end{array}$ & $3(1.6)$ & $11(0.4)$ & 27.3 & $2(2.5)$ & $4(0.9)$ & 50.0 & $1(0.9)$ & $7(0.3)$ & 14.3 \\
\hline $\begin{array}{l}\text { Moderate } \\
\text { hypoechogenicity }\end{array}$ & $28(14.6)$ & $109(4.3)$ & 25.7 & $17(21.0)$ & $37(8.6)$ & 45.9 & $11(9.9)$ & $72(3.4)$ & 15.3 \\
\hline $\begin{array}{l}\text { Mild } \\
\text { hypoechogenicity }\end{array}$ & $42(21.9)$ & $\begin{array}{l}334 \\
(13.0)\end{array}$ & 12.6 & $22(27.2)$ & $93(21.7)$ & 23.7 & $20(18.0)$ & $\begin{array}{l}241 \\
(11.3)\end{array}$ & 8.3 \\
\hline $\begin{array}{l}\text { Iso- or } \\
\text { hyperechogenicity }\end{array}$ & $119(62.0)$ & $\begin{array}{l}2106 \\
(82.3)\end{array}$ & 5.7 & $40(49.4)$ & $\begin{array}{l}294 \\
(68.7)\end{array}$ & 13.6 & 79 (71.2) & $\begin{array}{l}1812 \\
(85.0)\end{array}$ & 4.4 \\
\hline Total & $192(100.0)$ & $\begin{array}{l}2560 \\
(100.0)\end{array}$ & 7.5 & $81(100.0)$ & $\begin{array}{l}428 \\
(100.0)\end{array}$ & 18.9 & $111(100.0)$ & $\begin{array}{l}2132 \\
(100.0)\end{array}$ & 5.2 \\
\hline
\end{tabular}

When we categorized nodules according to composition and presence of suspicious features, there was no significant difference of malignancy risk between markedly and moderately hypoechoic nodules in all subgroups, regardless of composition and presence of suspicious feature $(P \geq 0.48)$. In solid nodules, markedly or moderately hypoechoic nodules showed significantly higher malignancy risk than mild hypoechoic $(P \leq 0.016)$ and iso- or hyperechoic $(P<$ .001) nodules regardless of suspicious features. 
In partially cystic nodules with suspicious features, moderately hypoechoic nodules showed significantly higher malignancy risk than mild hypoechoic $(P \leq$ $0.045)$ and iso- or hyperechoic nodules $(P<.001)$. There was no significant difference in malignancy risk in partially cystic nodules without suspicious features according to the degree of hypoechogenicity $(P \geq 0.116)$. Moderately $(P=0.008)$ and mildly hypoechoic $(P=0.017)$ nodules showed significantly higher malignancy risk than iso- or hyperechoic nodules in partially cystic nodules without suspicious features.

\section{Risk stratification of thyroid nodules according to the degree of hypoechogenicity}

In solid nodules, the malignancy risks of nodules with moderate (73.3\%) or marked hypoechogenicity (78.6\%) with suspicious features were within the high suspicion category. Solid nodules with mild hypoechogenicity and suspicious features showed a slightly lower malignancy risk than the lower margin of the high suspicion category (52.0\%). Solid, marked (31.3\%), moderate (25.9\%), and mild hypoechoic nodules (17.4\%) without suspicious features were stratified as intermediate risk.

In partially cystic nodules, marked (50.0\%) and moderate (45.9\%) hypoechogenicity showed slightly higher malignancy risks than the estimated range of the intermediate and low suspicion categories according to the presence of suspicious features. Partially cystic nodules with mild hypoechogenicity and iso- or hyperechogenicity were classified within the low and intermediate suspicion categories, according to the presence of suspicious features.

Comparisons of malignancy risks among four nodule groups, based on composition and echogenicity.

Table 4 illustrates the malignancy risks in the four groups of nodules categorized according to a combination of composition, predominant echogenicity, and presence of suspicious features. The malignancy risks differed significantly between these groups in the following decreasing order: solid hypoechoic, partially cystic hypoechoic, solid iso- or hyperechoic, and partially cystic iso- or hyperechoic. The malignancy risks significantly differed in all subgroups (all, $P<.001)$ except between partially cystic hypoechoic versus solid iso- or hyperechoic nodules $(P \geq 0.122)$, regardless of the presence of suspicious features

Table 4

Malignancy risk of four nodule categories based on composition and predominant echogenicity

\begin{tabular}{|c|c|c|c|c|c|c|c|c|}
\hline & $\begin{array}{l}\text { Solid } \\
\text { hypoechoic }\end{array}$ & & $\begin{array}{l}\text { Partially cystic } \\
\text { hypoechoic }\end{array}$ & & Solid isoechoic & & $\begin{array}{l}\text { Partially cystic } \\
\text { isoechoic }\end{array}$ & \\
\hline & $\begin{array}{l}\text { No. of } \\
\text { malignant } \\
\text { nodules/all }\end{array}$ & $\begin{array}{l}\text { Malignancy } \\
\text { risk (95\% Cl) }\end{array}$ & $\begin{array}{l}\text { No. of } \\
\text { malignant } \\
\text { nodules/all }\end{array}$ & $\begin{array}{l}\text { Malignancy } \\
\text { risk (95\% Cl) }\end{array}$ & $\begin{array}{l}\text { No. of } \\
\text { malignant } \\
\text { nodules/all }\end{array}$ & $\begin{array}{l}\text { Malignancy } \\
\text { risk (95\% Cl) }\end{array}$ & $\begin{array}{l}\text { No. of } \\
\text { malignant } \\
\text { nodules/all }\end{array}$ & $\begin{array}{l}\text { Malignancy } \\
\text { risk (95\% Cl) }\end{array}$ \\
\hline All & $705 / 1560$ & $\begin{array}{l}45.2(41.9 \\
48.7)\end{array}$ & $73 / 454$ & $\begin{array}{l}16.1(12.6 \\
20.2)\end{array}$ & $192 / 1481$ & $\begin{array}{l}13.0(11.2 \\
14.9)\end{array}$ & $119 / 2106$ & $5.7(4.7,6.8)$ \\
\hline$P$ & $<.001^{\mathrm{a}}$ & & $0.122^{b}$ & & & & $<.001^{\mathrm{c}}$ & \\
\hline $\begin{array}{l}\text { Any } \\
\text { suspicious } \\
\text { feature }\end{array}$ & $536 / 780$ & $\begin{array}{l}68.7(63.0 \\
74.8)\end{array}$ & $41 / 134$ & $\begin{array}{l}30.6(22.0 \\
41.5)\end{array}$ & $93 / 300$ & $\begin{array}{l}31.0(25.0, \\
38.0)\end{array}$ & $40 / 294$ & $\begin{array}{l}13.6(9.7 \\
18.5)\end{array}$ \\
\hline$P$ & $<.001^{\mathrm{a}}$ & & $0.954^{b}$ & & & & $<.001^{\mathrm{c}}$ & \\
\hline $\begin{array}{l}\text { No } \\
\text { suspicious } \\
\text { feature }\end{array}$ & $169 / 780$ & $\begin{array}{l}21.7(18.5, \\
25.2)\end{array}$ & $32 / 320$ & $\begin{array}{l}10.0(6.8 \\
14.1)\end{array}$ & $99 / 1181$ & $\begin{array}{l}8.4(6.8 \\
10.2)\end{array}$ & $79 / 1812$ & $4.4(3.5,5.4)$ \\
\hline$P$ & $<.001^{\mathrm{a}}$ & & $0.385^{\mathrm{b}}$ & & & & $<.001^{\mathrm{c}}$ & \\
\hline \multicolumn{9}{|c|}{ asolid hypoechoic vs. partially cystic hypoechoic } \\
\hline \multicolumn{9}{|c|}{${ }^{\text {b} P a r t i a l l y ~ c y s t i c ~ h y p o e c h o i c ~ v s . ~ S o l i d ~ i s o e c h o i c ~}$} \\
\hline
\end{tabular}

\section{Discussion}

Our study demonstrated no significant difference in malignancy risks between homogeneous vs. heterogeneous hypoechoic nodules in all subgroups and in homogenous vs. heterogeneous iso- or hyperechoic nodules in all subgroups except partially cystic nodules without suspicious features. Meanwhile, heterogeneous hypoechoic nodules showed significantly higher malignancy risk than heterogeneous isoechoic nodules in all subgroups except partially cystic nodules. Our study validated the concept of classifying nodules by their predominant echogenicity as a reasonable form of risk stratification. Regarding the degree of hypoechogenicity, nodules with moderate hypoechogenicity showed similar malignancy risks compared to markedly hypoechoic nodules. In contrast, moderately hypoechoic nodules showed significantly higher malignancy risks than mild hypoechoic nodules in all subgroups except partially cystic nodules without suspicious features. Based on our results, moderately hypoechoic nodules should be grouped with marked hypoechoic nodules for risk stratification.

For nodules with heterogeneous echogenicity, the EU-TIRADS suggested that nodules with any hypoechoic component should be regarded as hypoechoic nodules and classified as intermediate risk [10]. However, in our study, the malignancy risks of heterogeneous isoechoic nodules were not significantly different from their homogeneous counterparts except in the partially cystic nodules without suspicious features subgroup; this result aligned with the findings of our previous study [7]. Although the malignancy risks of heterogeneous isoechoic nodules were higher than homogeneous isoechoic nodules in 
overall nodules, the malignancy risks of heterogeneous isoechoic nodules were ranged within the low to intermediate risk categories, depending on concurrent suspicious US features. Therefore, our study's results support the strategy provided by K-TIRADS and ACR TIRADS when assessing nodules with heterogeneous echogenicity.

In the EU-TIRADS [10] and ACR-TIRADS [11], moderate hypoechogenicity was classified as similar risk with mild hypochogenicity; however in this study, moderate hypochogenicity showed a similar malignancy risk to marked hypochogenicity. The results of this study suggest that the previous definition of marked hypoechogenicity should be revised as hypoechoic or similar echogenicity relative to the anterior neck muscles. The result of this study confirmed the validity of the revised definition of marked hypoechogenicity by 2021 K-TIRADS.

The results of our study are in line with those of our previous study in that the malignancy risks of moderately hypoechoic nodules are similar to that of markedly hypoechoic nodules [7]. However, the results in overall nodules were somewhat discrepant from the previous study [7], demonstrating that the malignancy risks of marked hypoechoic nodules were higher than that of moderately hypoechoic nodules [6]. In this cohort, concurrent suspicious US features occurred more frequently in marked hypoechoic nodules than moderately hypoechoic nodules (marked hypoechoic, $72.4 \%$ vs. moderate hypoechoic, $52.5 \%, P<.001)$. The higher prevalence of suspicious features in marked hypoechoic nodules might have caused confounding effects in the malignancy risks between these two groups.

In the partially cystic nodules without suspicious features subgroup, the malignancy risks of most nodules ranged within the low risk category, regardless of their predominant echogenicity. This contrasts partially cystic nodules with suspicious features' risks, which fell within the intermediate risk category in most nodules. In contrast to solid nodules, partially cystic nodules without suspicious features showed no significant difference in malignancy risk between marked/moderate versus mild hypoechoic nodules, and the difference between various degrees of hypoechogenicity was diminished. Additionally, in partially cystic nodules, most partially cystic hypoechoic nodules showed mild hypoechogenicity (68.7-85.0\%) and the incidence of marked hypoechogenicity was very rare. Based on our results, we assume that in partially cystic nodules, malignancy risk is mainly determined by the presence of suspicious features and the degree of hypoechogenicity had little impact.

Our study has several limitations. First, the reference standards for benign and malignant diagnoses were based on FNA results as well as surgical histology findings, which may inevitably cause false-negative or false-positive results, although the effect may have been small. Second, we retrospectively assessed the nodules' US features. However, we believe our large sample size across a multicenter setting mitigated this shortcoming. Third, this study did not consider the interobserver agreement for US features described by different radiologists, possibly resulting in discrepant interpretations. However, we performed training sessions prior to formal analysis to mitigate for interobserver variability. Although a previous study demonstrated that this proposed classification of echogenicity showed improved reproducibility than the previous lexicon [7], future in-depth studies are needed to validate the reproducibility of this US lexicon in multiple readers.

In conclusion, the malignancy risk of nodules with heterogeneous echotexture can be stratified based on predominant echogenicity. Additionally, nodule hypoechogenicity can be classified as mild vs. moderate to marked hypoechogenicity for malignancy risk stratification.

\section{Declarations}

Funding This research was supported by research fund from the Korean Society of Radiology through Radiology Imaging Network of Korea for Clinical Research (RINK-CR).

Conflict of Interest The authors declare no competing interest.

Author contributions Conceptualization: J.Y. Lee, C.Y. Lee, D.G. Na; Data Curation: all authors; Methodology: J.Y. Lee, C.Y. Lee, D. G. Na; Project administration: J.Y.Lee, C.Y. Lee, J-h. Kim, D.G. Na; Formal analysis: all authors; Investigation: all authors; Resources: J.Y. Lee, C.Y. Lee, J-h. Kim, D.G. Na; Supervision: all authors; Writing-original draft preparation: J.Y. Lee.; Writing-review and editing: D. G. Na; Funding acquisition: D. G. Na, J-h. Kim

Ethics approva/ This retrospective study was approved by the institutional review boards of 26 participating centers.

Consent to participate/Consent to publish The requirement for patient informed consent was waived

\section{References}

1. W.J. Moon, S.L. Jung, J.H. Lee, D.G. Na, J.H. Baek, Y.H. Lee, J. Kim, H.S. Kim, J.S. Byun, D.H. Lee et al.: Benign and malignant thyroid nodules: US differentiation--multicenter retrospective study. Radiology, 247(3):762-770(2008)10.1148/radiol.2473070944

2. P. Campanella, F. Ianni, C.A. Rota, S.M. Corsello, A. Pontecorvi: Quantification of cancer risk of each clinical and ultrasonographic suspicious feature of thyroid nodules: a systematic review and meta-analysis. European journal of endocrinology, 170(5):R203-211(2014)10.1530/eje-13-0995

3. J.P. Brito, M.R. Gionfriddo, A. Al Nofal, K.R. Boehmer, A.L. Leppin, C. Reading, M. Callstrom, T.A. Elraiyah, L.J. Prokop, M.N. Stan et al.: The accuracy of thyroid nodule ultrasound to predict thyroid cancer: systematic review and meta-analysis. The Journal of clinical endocrinology and metabolism, 99(4):1253-1263(2014)10.1210/jc.2013-2928

4. D.G. Na, J.H. Baek, J.Y. Sung, J.H. Kim, J.K. Kim, Y.J. Choi, H. Seo: Thyroid Imaging Reporting and Data System Risk Stratification of Thyroid Nodules: Categorization Based on Solidity and Echogenicity. Thyroid, 26(4):562-572(2016)10.1089/thy.2015.0460 
5. J.H. Kim, D.G. Na, H. Lee: Ultrasonographic Echogenicity and Histopathologic Correlation of Thyroid Nodules in Core Needle Biopsy Specimens. Korean J Radiol, 19(4):673-681(2018)10.3348/kjr.2018.19.4.673

6. M.H. Wu, C.N. Chen, K.Y. Chen, M.C. Ho, H.C. Tai, Y.H. Wang, A. Chen, K.J. Chang: Quantitative analysis of echogenicity for patients with thyroid nodules. Sci Rep, 6:35632(2016)10.1038/srep35632

7. J.Y. Lee, D.G. Na, S.J. Yoon, H.Y. Gwon, W. Paik, T. Kim, J.Y. Kim: Ultrasound malignancy risk stratification of thyroid nodules based on the degree of hypoechogenicity and echotexture. Eur Radiol, 30(3):1653-1663(2020)10.1007/s00330-019-06527-8

8. R.L.C. Delfim, L. Veiga, A.P.A. Vidal, F. Lopes, M. Vaisman, P. Teixeira: Likelihood of malignancy in thyroid nodules according to a proposed Thyroid Imaging Reporting and Data System (TI-RADS) classification merging suspicious and benign ultrasound features. Arch Endocrinol Metab, 61(3):211221(2017)10.1590/2359-3997000000262

9. H. Gharib, E. Papini, J.R. Garber, D.S. Duick, R.M. Harrell, L. Hegedüs, R. Paschke, R. Valcavi, P. Vitti: AMERICAN ASSOCIATION OF CLINICAL ENDOCRINOLOGISTS, AMERICAN COLLEGE OF ENDOCRINOLOGY, AND ASSOCIAZIONE MEDICI ENDOCRINOLOGI MEDICAL GUIDELINES FOR CLINICAL PRACTICE FOR THE DIAGNOSIS AND MANAGEMENT OF THYROID NODULES - 2016 UPDATE. 22(Supplement 1):1-60(2016)10.4158/ep161208.GI

10. G. Russ, S.J. Bonnema, M.F. Erdogan, C. Durante, R. Ngu, L. Leenhardt: European Thyroid Association Guidelines for Ultrasound Malignancy Risk Stratification of Thyroid Nodules in Adults: The EU-TIRADS. Eur Thyroid J, 6(5):225-237(2017)10.1159/000478927

11. F.N. Tessler, W.D. Middleton, E.G. Grant, J.K. Hoang, L.L. Berland, S.A. Teefey, J.J. Cronan, M.D. Beland, T.S. Desser, M.C. Frates et al.: ACR Thyroid Imaging, Reporting and Data System (TI-RADS): White Paper of the ACR TI-RADS Committee. J Am Coll Radiol, 14(5):587-

595(2017)10.1016/j.jacr.2017.01.046

12. J. Zhou, L. Yin, X. Wei, S. Zhang, Y. Song, B. Luo, J. Li, L. Qian, L. Cui, W. Chen et al.: 2020 Chinese guidelines for ultrasound malignancy risk stratification of thyroid nodules: the C-TIRADS. Endocrine, 70(2):256-279(2020)10.1007/s12020-020-02441-y

13. S.J. Yoon, D.G. Na, H.Y. Gwon, W. Paik, W.J. Kim, J.S. Song, M.S. Shim: Similarities and Differences Between Thyroid Imaging Reporting and Data Systems. American Journal of Roentgenology, 213(2):W76-W84(2019)10.2214/AJR.18.20510

14. J.H. Shin, J.H. Baek, J. Chung, E.J. Ha, J.H. Kim, Y.H. Lee, H.K. Lim, W.J. Moon, D.G. Na, J.S. Park et al.: Ultrasonography Diagnosis and Imaging-Based Management of Thyroid Nodules: Revised Korean Society of Thyroid Radiology Consensus Statement and Recommendations. Korean J Radiol, 17(3):370-395(2016)10.3348/kjr.2016.17.3.370

15. B.R. Haugen, E.K. Alexander, K.C. Bible, G.M. Doherty, S.J. Mandel, Y.E. Nikiforov, F. Pacini, G.W. Randolph, A.M. Sawka, M. Schlumberger et al.: 2015 American Thyroid Association Management Guidelines for Adult Patients with Thyroid Nodules and Differentiated Thyroid Cancer: The American Thyroid Association Guidelines Task Force on Thyroid Nodules and Differentiated Thyroid Cancer. Thyroid, 26(1):1-133(2016)10.1089/thy.2015.0020

16. E.J. Ha, S.R. Chung, D.G. Na, H.S. Ahn, J. Chung, J.Y. Lee, J.S. Park, R.-E. Yoo, J.H. Baek, S.M. Baek et al., 2021 Korean Thyroid Imaging Reporting and Data System and Imaging-Based Management of Thyroid Nodules: Korean Society of Thyroid Radiology Consensus Statement and Recommendations. Korean J Radiol 22, 0 (2021)

17. E.J. Ha, J.H. Shin, D.G. Na, S.L. Jung, Y.H. Lee, W. Paik, M.J. Hong, Y.K. Kim, C.Y. Lee: Comparison of the diagnostic performance of the modified Korean Thyroid Imaging Reporting and Data System for thyroid malignancy with three international guidelines. Ultrasonography(2021)10.14366/usg.21056

18. S.R. Chung, H.S. Ahn, Y.J. Choi, J.Y. Lee, R.E. Yoo, Y.J. Lee, J.Y. Kim, J.Y. Sung, J.H. Kim, J.H. Baek: Diagnostic Performance of the Modified Korean Thyroid Imaging Reporting and Data System for Thyroid Malignancy: A Multicenter Validation Study. Korean J Radio/(2021)10.3348/kjr.2021.0230

\section{Figures}


22775 consecutive patients underwent thyroid US from 26 institutions from June to September 2015

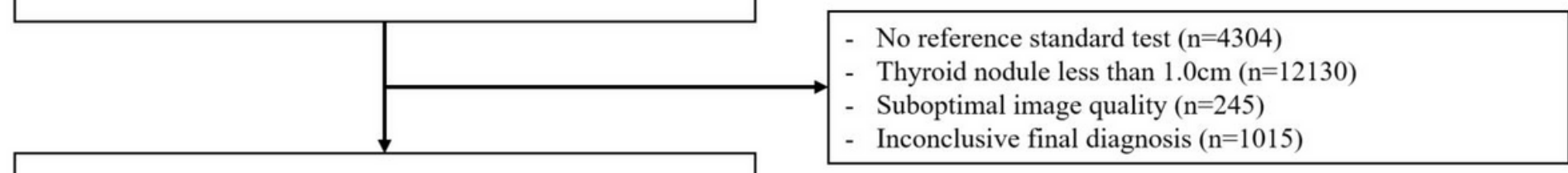

5708 thyroid nodules in 5081 patients

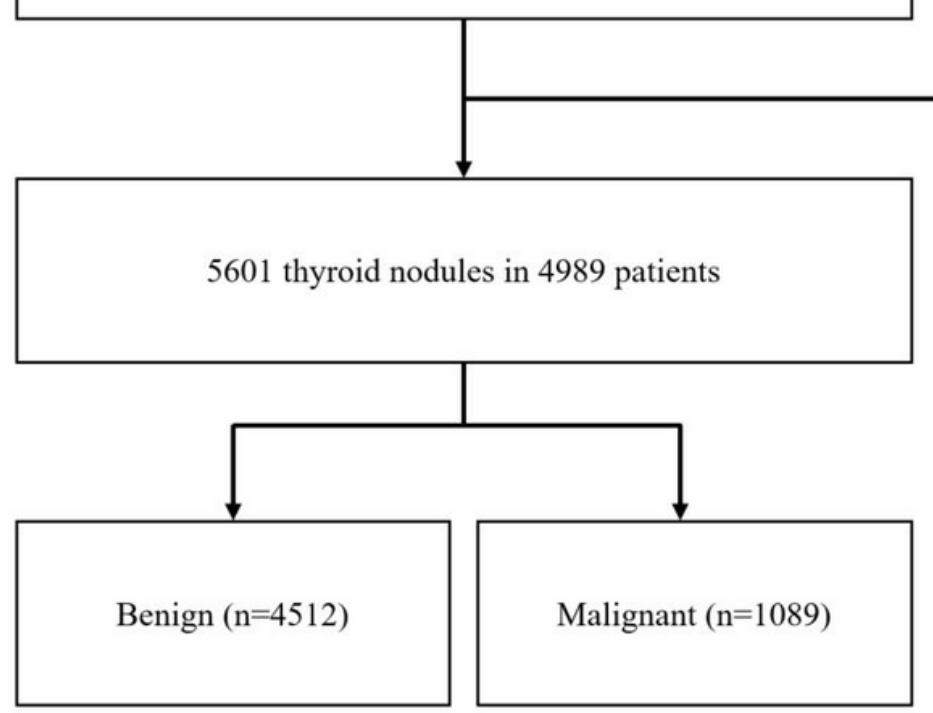

- Isolated macrocalcifications $(\mathrm{n}=59)$

- Purely cystic nodules $(n=48)$

Figure 1

Flowchart of the study. US=ultrasonography
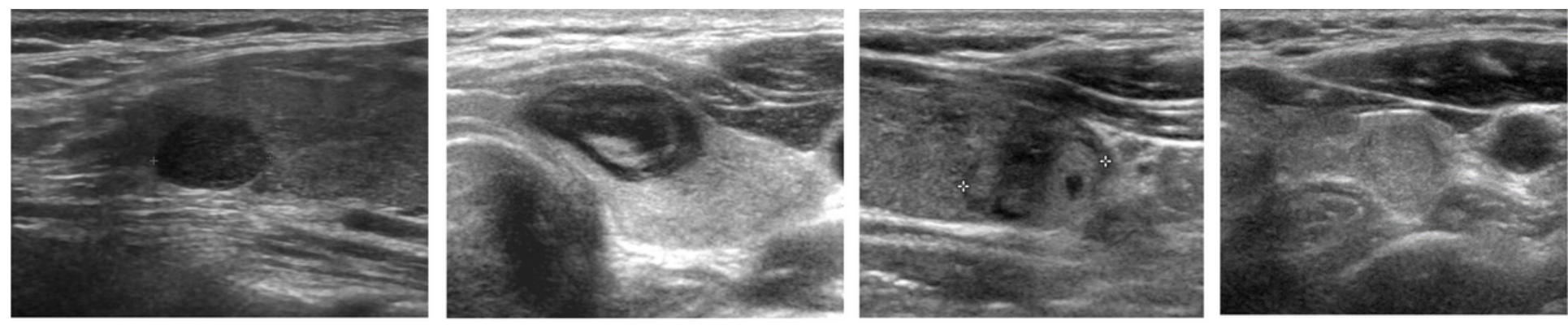

Figure 2

Thyroid nodules classified according to the echotexture and echogenicity. A. A nodule with homogeneous hypoechogenicity. Diagnosis: Conventional papillary thyroid carcinoma B. A nodule with heterogeneous, predominant hypoechogenicity. Note internal iso- or hyperechoic solid portions consisting less than $50 \%$ of the nodule. Diagnosis: Conventional papillary thyroid carcinoma. C. Nodule with heterogeneous, predominant iso- or hyperechogenicity. The hypoechoic solid portion accounts for less than $50 \%$ of the nodule. Diagnosis: Benign follicular nodule in core needle biopsy. D. Nodule with homogeneous iso- or hyperechogenicity. Diagnosis: Benign follicular nodule in core needle biopsy 

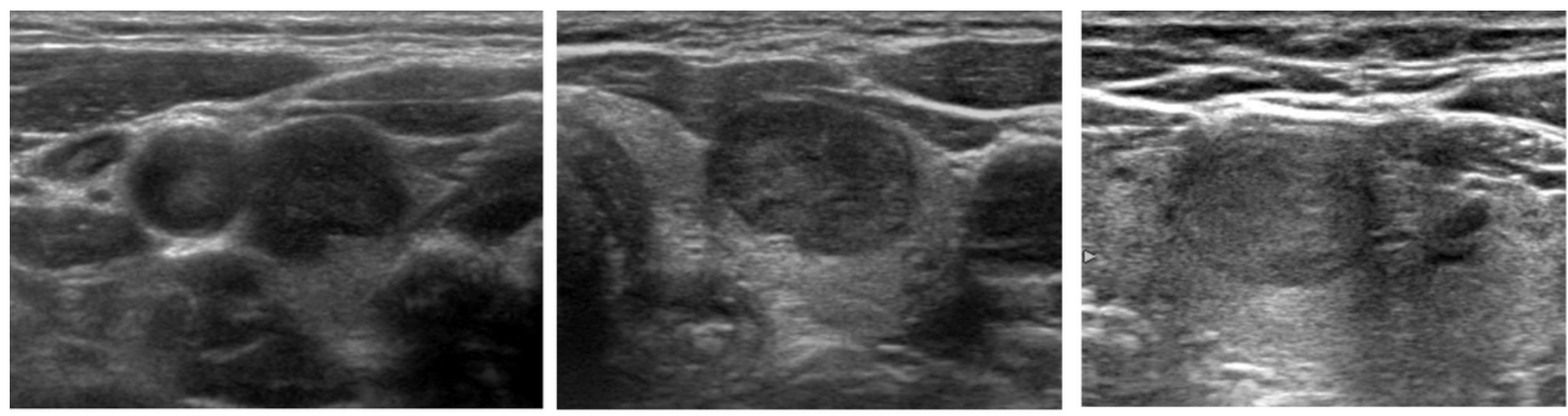

\section{Figure 3}

Hypoechoic thyroid nodules with various degree of hypoechogenicity. A. Markedly hypoechoic nodule (hypoechoic relative to the anterior neck muscles) Diagnosis: Conventional papillary thyroid carcinoma B. Moderately hypoechoic nodule (similar echogenicity to the anterior neck muscle). Diagnosis: Conventional papillary thyroid carcinoma C. Mildly hypoechoic nodule (hypoechoic relative to the normal thyroid parenchyma, but hyperechoic relative to the anterior neck muscle). Diagnosis: Follicular variant papillary thyroid carcinoma 